\title{
Numerical Minimum Time Control to a Class of Singular Integro-Differential Equations
}

\author{
Shihchung Chiang \\ Department of Finance, Chung Hua University, Hsinchu, Taiwan
}

Received: November 24, 2014 / Accepted: December 20, 2014 / Published: February 25, 2015.

\begin{abstract}
This study presents a numerical method for determining the minimum time required for the states of one class of integro-differential equations of the first kind to reach its attainable region by assuming the forcing terms of the equations as controls. These equations consist of integro-differential parts containing weakly singular kernels. The feasibility of the numerical method is demonstrated by comparing the minimum time and corresponding possible time by using extreme controls to reach the attainable region under different initial conditions.
\end{abstract}

Key words: Integro-differential equations, weakly singular kernels, minimum time control

\section{Introduction}

This study presents a numerical method for determining the minimum time required for the states of a class of singular integro-differential equations to reach an attainable region. Controls are assumed to be bounded. This class of equations originates from the aeroelasticity problem, and consists of an integro-differential term $[1,2,5,10]$. Under an integrable assumption, the equations can be transformed into Volterra integral equations, and accurate solutions can thus be obtained [2, 3, 4]. Based on previous studies $[6,7,8]$, this study assumed that the forcing terms of the equations are the control forces. The remainder of this study is organized as follows: Section 2 presents the weakly singular type of integro-differential equations. Section 3 describes the setting and numerical method. Section 4 presents the numerical results, especially the minmum time required to reach the possible attainable region. The numerical results also include the minimum time required to reach a specific point and the behavior of

Corresponding author: Shihchung Chiang, Department of Finance, Chung Hua University, Hsinchu, Taiwan, Email: Chiang@chu.edu.tw. the corresponding optimal state. Finally, Section 5 offers a summary of this study.

\section{Original Model}

Consider the class of singular integro-differential equations

$$
\frac{d}{d t} D x_{t}=u(t)
$$

with initial data

$$
x(s)=\phi(s), \quad-b \leq s \leq 0,
$$

where $b$ is a positive number. The difference operator $D$ is defined as

$$
D x_{t}=\int_{-b}^{0} x_{t}(s) g(s) d s,
$$

and the first part of the integrand represents

$$
x_{t}(s)=x(t+s) .
$$

The secong part is a weighting function

$$
g(s) \in L_{1}[-b, 0],
$$

which is integrable, positive, nondecreasing, and weakly singular at $s=0$. The control term $u(t)$ is locally integrable for $t>0$. Although a more general kernel $g$ is also effective, this study focuses on the Abel type of kernel (i.e., $g(s)=|s|^{-p}$, where 
$s \in[-b, 0])$ with $p=0.5$ based on the original aeroelastic problem.

The initial condition $\phi(s),-b \leq s \leq 0$, is in $L_{1, g}$, a weighted $L_{1}$ space with weight $g(\cdot)$. Note that for the initial value problems, Equations (1)-(2) can be expressed as

$$
D x_{t}=D x_{0}+\int_{0}^{t} f(\tau) d \tau,
$$

provided that the function

$$
D x_{t}=\int_{-b}^{0} g(s) x(t+s) d s
$$

is absolutely continuous for $t>0$, and the function $g(\cdot) \phi(\cdot)$ belongs to $L_{1}[-b, 0]$. Without loss of generality, assume that $b=1$.

\section{Numerical Method}

This study considers a typical cost function:

$$
\Phi=T,
$$

where $T$ is the time for the state to reach attainable regions by using bounded controls $u(t)$. In this study, controls $u(t)$ satisfy $|u(t)| \leq 1$, for $t>0$. The procedure for determining the minimum time required to reach attainable set involves discretizing Equation (1) initially, and then constructing a linear system with unknowns used as states and controls. The space mesh points (corresponding to $s$ variable) are $-1=\tau_{n}$ $<\tau_{n-1}<\cdots<\tau_{1}<\tau_{0}=0$, and a new variable $\xi$ is defined as

$$
\xi(t, s)=x(t+s), \quad-1 \leq s \leq 0, \quad t>0 .
$$

Equation (1) can then be reformulated as a first-order hyperbolic equation [9] :

$$
\frac{\partial}{\partial t} \xi(t, s)=\frac{\partial}{\partial s} \xi(t, s), \quad-1 \leq s \leq 0,
$$

under the condition

$$
\int_{-1}^{0}|s|^{-0.5} \frac{\partial}{\partial s} \xi(t, s) d s=u(t) .
$$

Next, assume that the solutions to Equations (4)- (5) have the form

$$
\xi(t, s)=\sum_{i=0}^{n} \alpha_{i}(t) B_{i}(s),
$$

where the basis, $B_{i}(s), i=0, \cdots, n$, is given by

$$
B_{i}(s)= \begin{cases}\frac{1}{\left(\tau_{i}-\tau_{i+1}\right)}\left(s-\tau_{i+1}\right) & s \in\left[\tau_{i+1}, \tau_{i}\right], \\ \frac{1}{\left(\tau_{i-1}-\tau_{i}\right)}\left(\tau_{i-1}-s\right) & s \in\left[\tau_{i}, \tau_{i-1}\right], \\ 0 & \text { otherwise . }\end{cases}
$$

In other words, $B_{i}(s), \quad i=0,1, \cdots, n$, are piecewise linear functions. After substituting the special form of $\xi$ in Equation (6) into Equations (4)-(5), the governing equations for $\alpha_{i}(t), \quad i=0, \cdots, n$, become the following semi-discretization system:

$$
\begin{aligned}
& \frac{d}{d t} \alpha_{i}(t)=\frac{1}{\delta_{i}}\left(\alpha_{i-1}(t)-\alpha_{i}(t)\right), i=1, \cdots, n, \\
& \int_{-1}^{0}|s|^{-p} \sum_{i=0}^{n} \alpha_{i}(t) \frac{d}{d s} B_{i}(s) d s=u(t),
\end{aligned}
$$

where $\delta_{i}=\tau_{i-1}-\tau_{i}>0$, for $i=1, \cdots, n$. For the full-discretization system, the discretization of time $t$ contains $T^{0}, T^{1}, \cdots, T^{m}$, where $0=T^{0}<T^{1}<\cdots$ $<T^{m}=1$. By defining $\Delta^{k}=T^{k+1}-T^{k}$, for $k=0, \cdots, m-1$, Equations (7)-(8) can then be expressed as

$$
\frac{1}{\Delta^{k}}\left(\alpha_{i}^{k+1}-\alpha_{i}^{k}\right)=\frac{1}{\delta_{i}}\left(\alpha_{i-1}^{k}-\alpha_{i}^{k}\right)
$$

and

$$
\sum_{i=1}^{n} \frac{g_{i}}{\delta_{i}}\left(\alpha_{i-1}^{k+1}-\alpha_{i}^{k+1}\right)=u\left(T^{k+1}\right),
$$

for $i=1, \ldots, n, \quad k=0, \ldots, m-1$, and $g_{i}=\int_{\tau_{i}}^{\tau_{i-1}}|s|^{-p} d s$.

Furthermore, assuming uniform mesh for both time and space, then the mesh points $\tau_{i}, \quad i=0, \cdots, n$, and $T^{k}, \quad k=0, \cdots, m$, are $\tau_{i}=-\frac{i}{n}, \quad T^{k}=\frac{k}{m}$, for some positive integers $n$ and $m$. The associated differences are defined as $\Delta^{k}=T^{k+1}-T^{k}$, $k=0, \cdots, m-1$, and $\delta_{i}=\tau_{i-1}-\tau_{i}, \quad i=1, \cdots, n ; \quad$ thus, $\Delta^{k}=1 / m$ and $\delta_{i}=1 / n, \quad$ for $k=0, \cdots, m-1$, and $i=1, \ldots, n$. Furthermore, setting $m=n$ produces the relationship $\Delta^{k}=\delta_{i}=1 / n$ for $k=0, \cdots, n-1$, and $i=1, \cdots, n$. Then, Equations (9)-(10) then lead to the following system: 


$$
\alpha_{i}^{k+1}=\alpha_{i-1}^{k}
$$

for $i=1, \cdots, n, \quad k=0, \cdots, n-1$, and

$$
\begin{gathered}
\sum_{i=1}^{n} \frac{1}{\delta_{i}}\left(\alpha_{i-1}^{k+1}-\alpha_{i}^{k+1}\right) \cdot \frac{1}{1-p}\left[-\left(-\tau_{i-1}\right)^{1-p}+\left(-\tau_{i}\right)^{1-p}\right] \\
=u\left(T^{k+1}\right)=u_{k+1},
\end{gathered}
$$

for $i=1, \cdots, n$, and $k=0, \cdots, n-1$.

After establishing constants $c_{0}, c_{1}, \cdots, c_{n}$, Equation (12) can be more simply in the following form :

$$
\alpha_{0}^{k+1} c_{0}+\alpha_{0}^{k} c_{1}+\cdots+\alpha_{0}^{0} c_{k+1}+\cdots+\alpha_{n-k-1}^{0} c_{n}=u_{k+1}
$$

$k=0, \cdots, n-1$. (13) The connection of the solution $x(t)$ and $\alpha$ 's is as follows :

Because $\xi(t, s)=x(t+s)$, for $-1 \leq s \leq 0, \quad t>0$, and $\xi(t, s)=\sum_{i=0}^{n} \alpha_{i}(t) B_{i}(s)$. Therefore $x(\bar{t})$, for $\bar{t}>0$, can be obtained in two cases :

Case $1($ fix $t=1)$ :

$$
x\left(1+\tau_{i}\right)=\sum_{l=0}^{n} \alpha_{l}(1) B_{l}\left(\tau_{i}\right)=\alpha_{i}(1)=\alpha_{i}^{n},
$$

Case $2($ fix $s=0)$ :

$$
x\left(T^{j}\right)=\sum_{l=0}^{n} \alpha_{l}\left(T^{j}\right) B_{l}(0)=\alpha_{0}\left(T^{j}\right)=\alpha_{0}^{j} .
$$

Because each time step used in Equation (13) is $\Delta^{k}=1 / n$, for $k=0, \cdots, n-1$, a system of equations can be constructed as follows:

$$
\begin{gathered}
\alpha_{0}^{1} c_{0}+\alpha_{0}^{0} c_{1}+\cdots+\alpha_{n-1}^{0} c_{n}=u_{1}, \\
\alpha_{0}^{2} c_{0}+\alpha_{0}^{1} c_{1}+\alpha_{0}^{0} c_{2}+\cdots+\alpha_{n-2}^{0}=u_{2}, \\
\vdots \\
\alpha_{0}^{n} c_{0}+\alpha_{0}^{n-1} c_{1}+\alpha_{0}^{n-2} c_{2}+\cdots+\alpha_{0}^{0} c_{n}=u_{n} .
\end{gathered}
$$

In System (14), the unknowns are states $\alpha_{0}^{k}$ and controls $u_{k}$, for $k=1,2, \cdots, n$. The values of states $\alpha_{i}^{0}, i=0,1, \cdots, n-1$, can be obtained based on the initial condition.

The attainable region for Equations (1)-(2) is obtained by setting the controls equal to the extreme values : $\quad u_{k}=1, k=1,2, \cdots, n$, for the first direction, and $u_{k}=-1, \quad k=1,2, \cdots, n, \quad$ for the second direction. Apply extreme controls to the linear System (14), the unknowns $\alpha_{0}^{i}, i=1,2, \cdots, n$, are solved. After completely solving System (14), the time step moves forward by a step length $1 / n$, and the already solved states $\alpha_{0}^{i}, i=1,2, \cdots, n$, are collected and set as the initial conditions for the next iteration. This type of process repeats until the final time is reached.

In this moving process, to avoid applying an inappropriate control force, the suggested tolerance for the control to change from one extreme value to another is the difference of two consecutive states being greater than 0.1 and the orientation moving backward to the initial state.

Regarding the minimum time, the state of Equations (1)-(2) tends to move in a positive direction when the control force is positive, and moves in a negative direction when the control force is negative. Because of these phenomena, after establishing the final goal for the state to achieve, determining the control in the first time step is necessary to consider the relative positions of the initial condition $\alpha_{0}^{0}$ and the final goal for choosing a suitable control force.

The criterion for the state to reach the final goal is when the distance between the state and the final goal being less than 0.1 in the moving process. The condition for the corresponding optimal controls to change from one extreme value to another is also determined according to the difference between two consecutive states. If the difference is greater than 0.1 and the orientation of the state is not moving toward the final goal, then a change of control is executed.

\section{Numerical Examples}

In the following examples, the maximum final time required to reach an attainable region is limited to be 20 units, and two figures are presented for each example with $n=100$. The first figure displays two curves: the first curve presents the time consumed vs. the reachable set $[-10,10]$, and the second curve is 
the minimum time required vs. the reachable set $[-10,10]$. The second figure also depicts two curves: the first curve represents the same graph of the minimum time with respect to the reachable set, and the second curve shows the optimal state vs. the time required to reach a typical final goal of -4 .

\section{Example 1.}

Initial condition $\phi(s)=0,-1 \leq s \leq 0$. The results are shown in Figures 3.1 and 3.2.

\section{Example 2.}

Initial condition $\phi(s)=1, \quad-1 \leq s \leq 0$. The results are presented in Figures 3.3 and 3.4.

\section{Example 3.}

Initial condition $\phi(s)=-1,-1 \leq s \leq 0$. The results are shown in Figures 3.5 and 3.6.

\section{Example 4.}

Initial condition $\phi(s)=-s,-1 \leq s \leq 0$. The results are presented in Figures 3.7 and 3.8.

\section{Example 5.}

Initial condition $\phi(s)=s,-1 \leq s \leq 0$. The results are shown in Figures 3.9 and 3.10.

\section{Example 6.}

Initial condition $\phi(s)=\sin (s), \quad-1 \leq s \leq 0$. The results are presented in Figures 3.11 and 3.12.

\section{Example 7.}

Initial condition $\phi(s)=\cos (s), \quad-1 \leq s \leq 0 . \quad$ The results are shown in Figures 3.13 and 3.14.

\section{Summary}

This study presents a numerical method and the results of determining the minimum time and corresponding optimal state to reach the attainable set under different initial conditions for solving minimum time problem, and a scalar singular integro-differential equation is the constraint. The criterion for the state to reach the final goal is when the distance in between is less than 0.1 .

This study demonstrates the feasibility of the proposed method by comparing the minimum time vs. final goal and time vs. attainable region, starting from different initial conditions. This study presents the corresponding optimal state to reach the final goal of -4 in each example.

\section{Appendix}

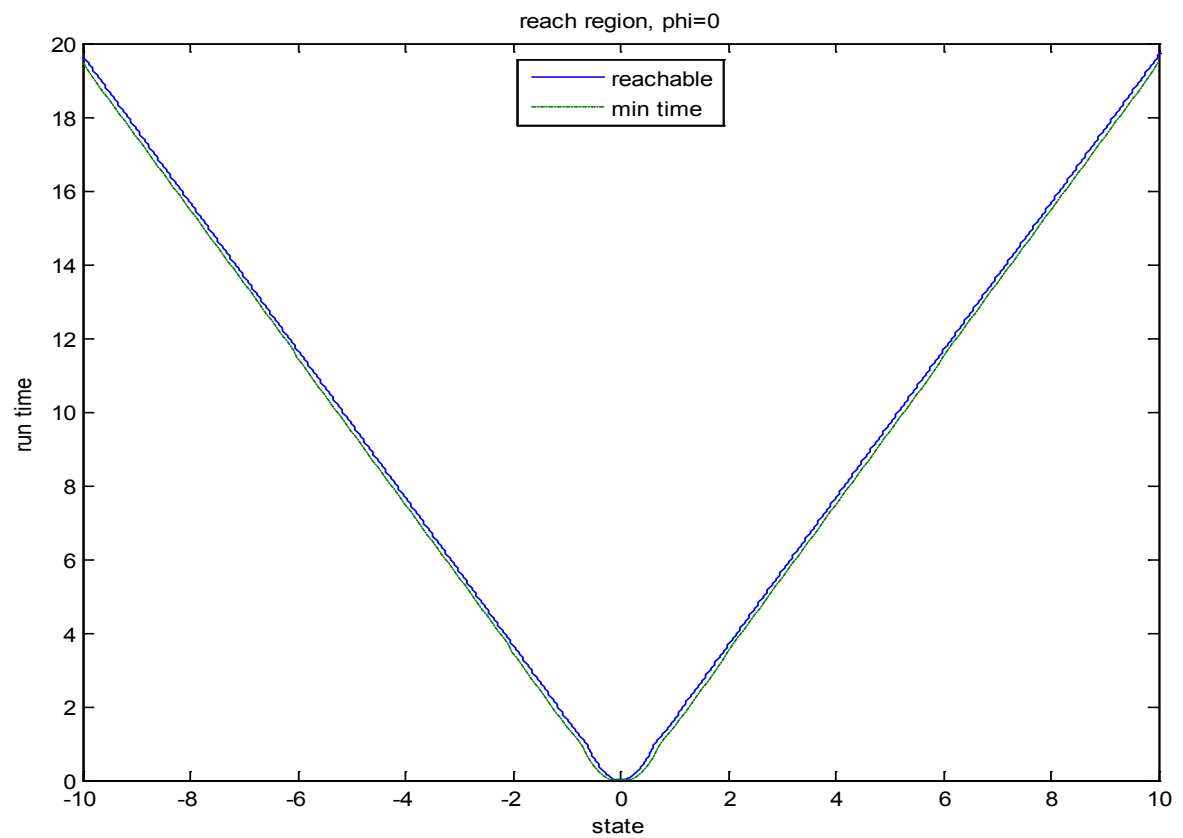

Figures 3.1 

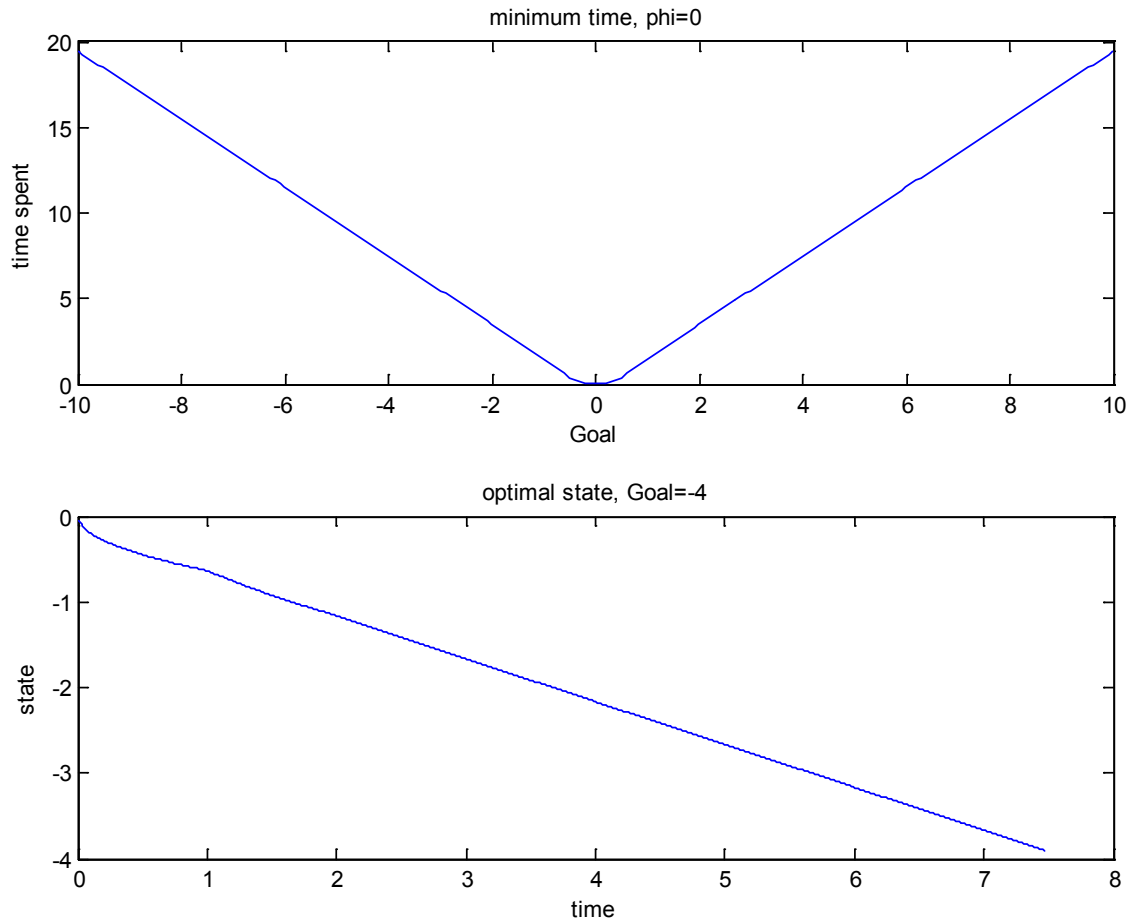

Figure 3.2

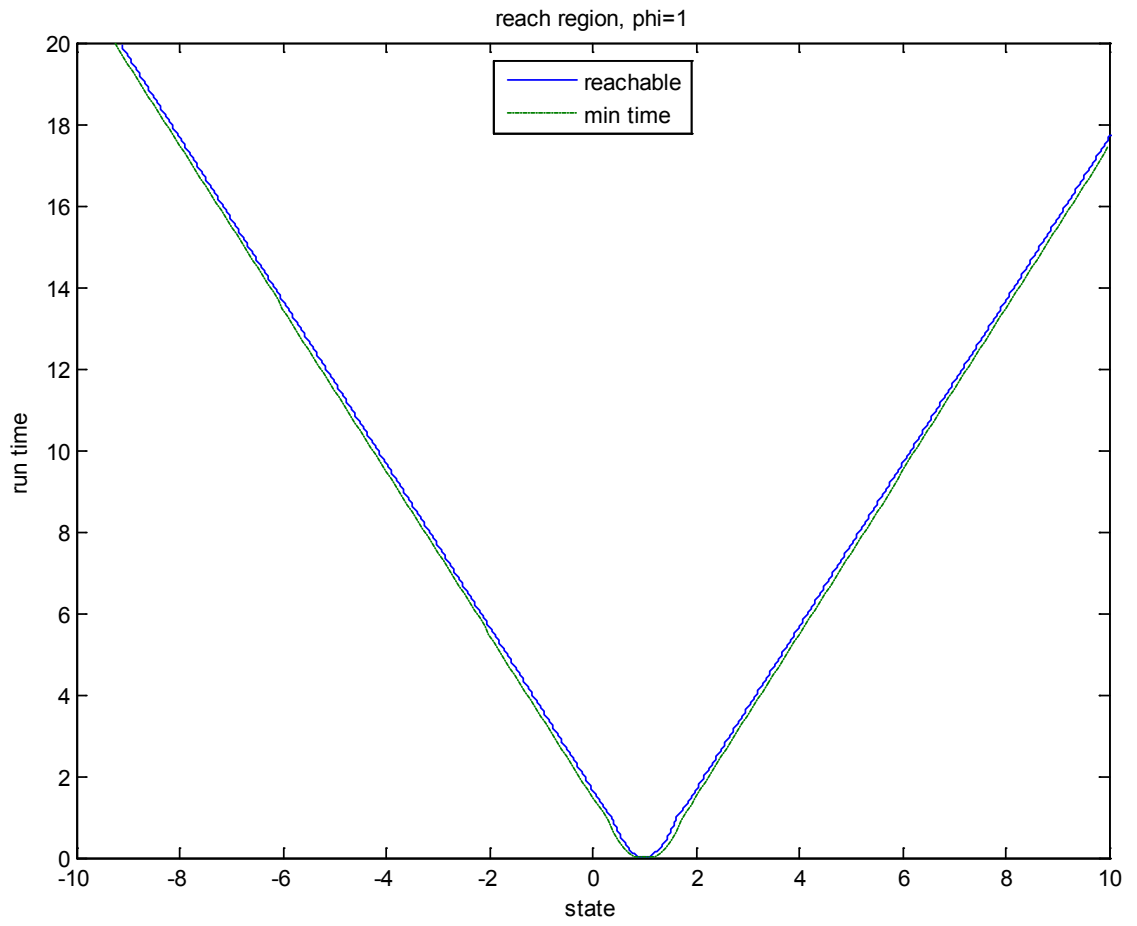

Figure 3.3 

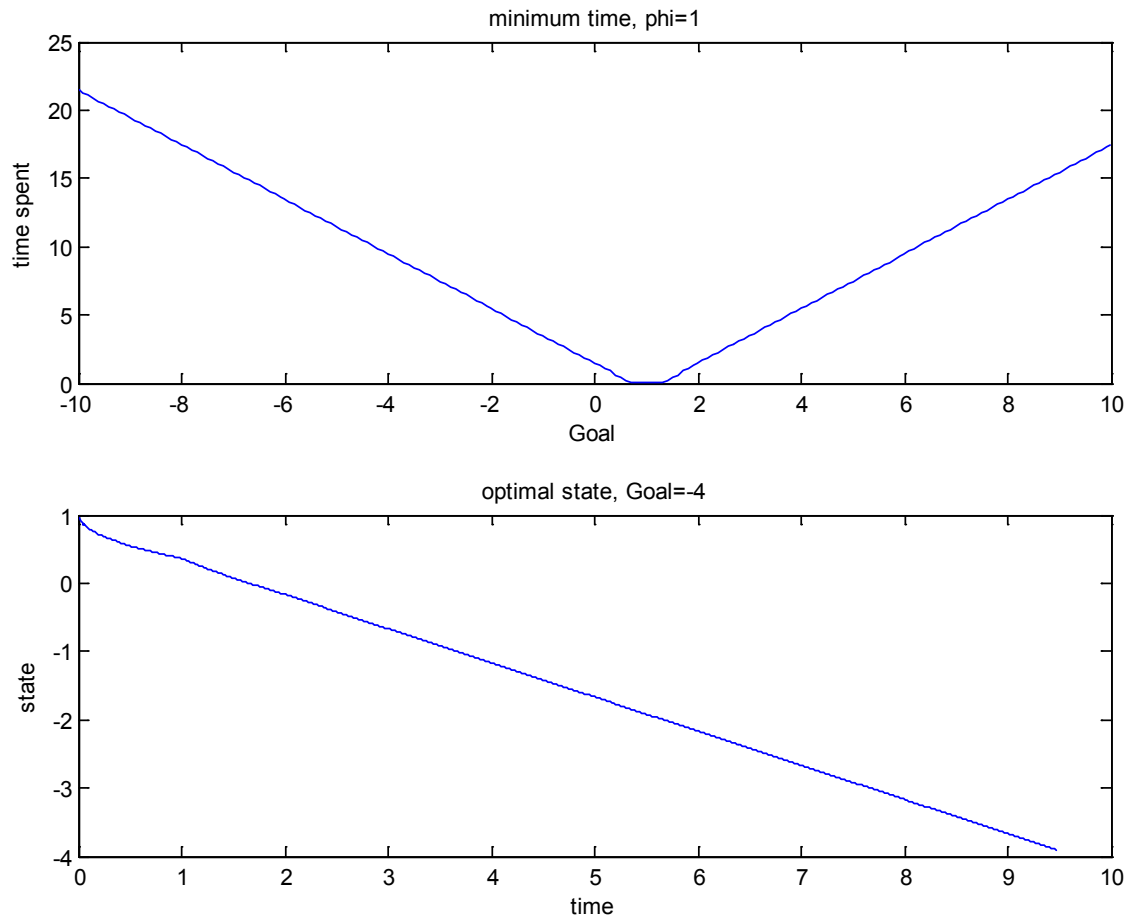

Figure 3.4

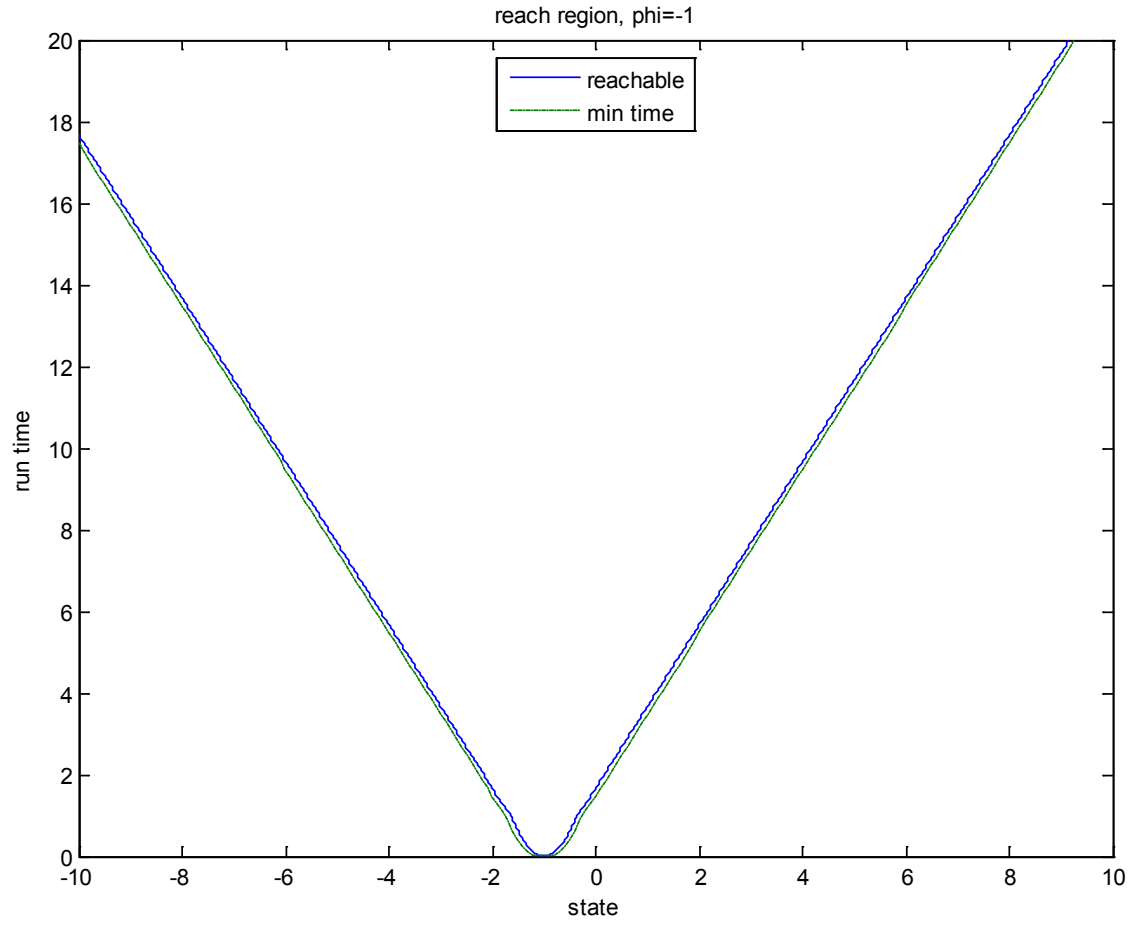

Figure 3.5 

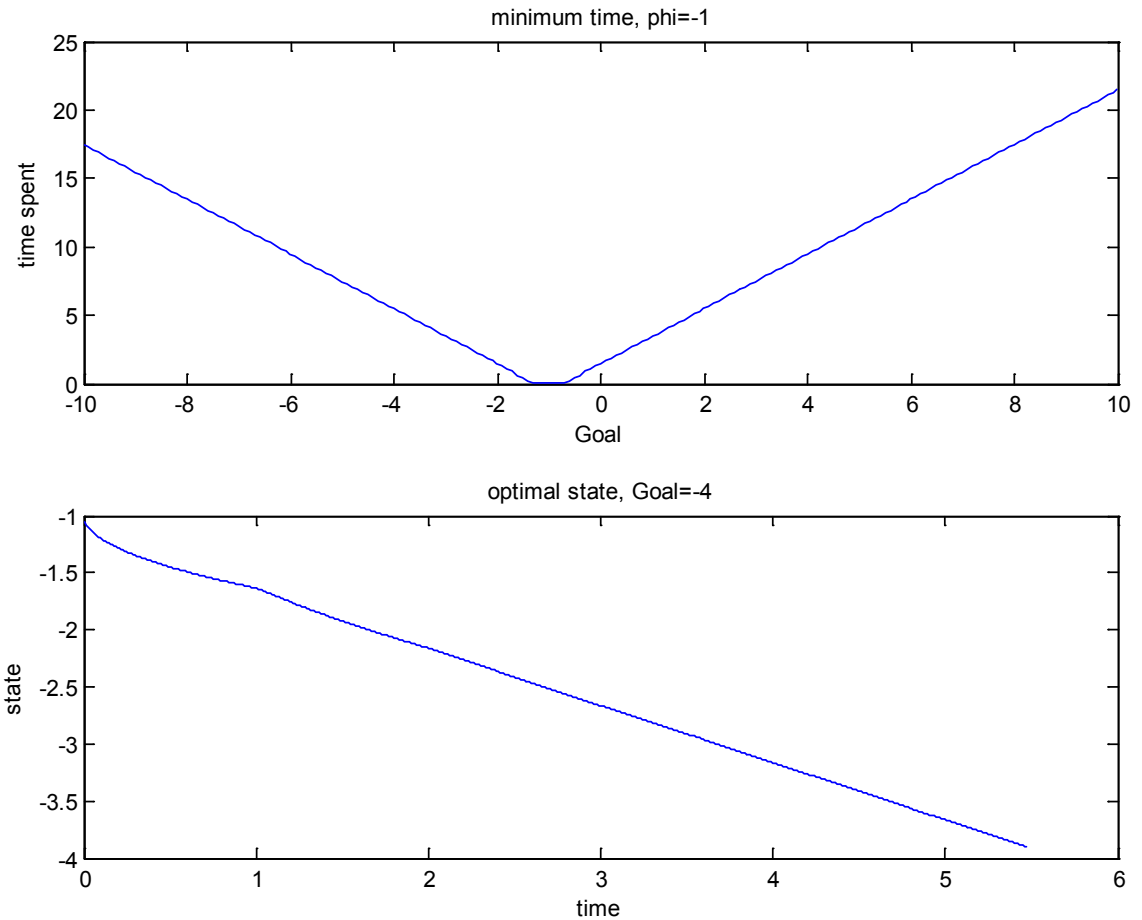

Figure 3.6

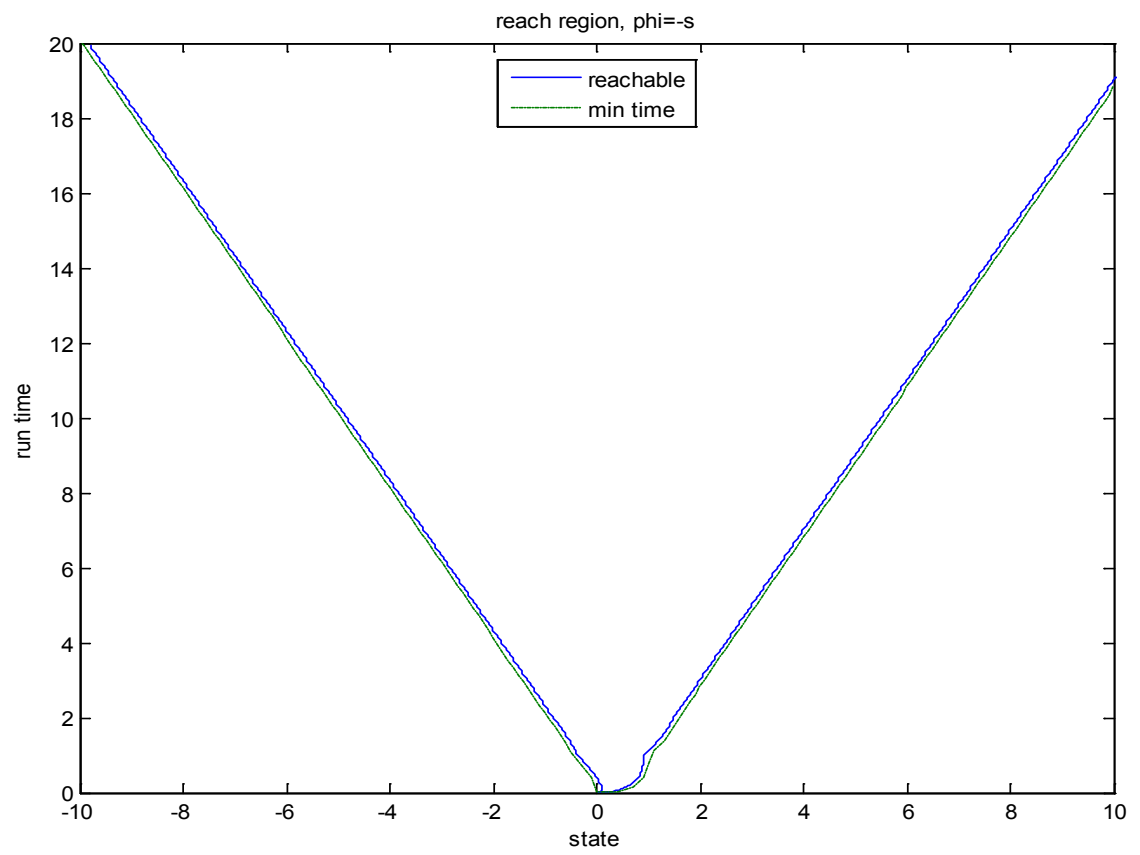

Figure 3.7 

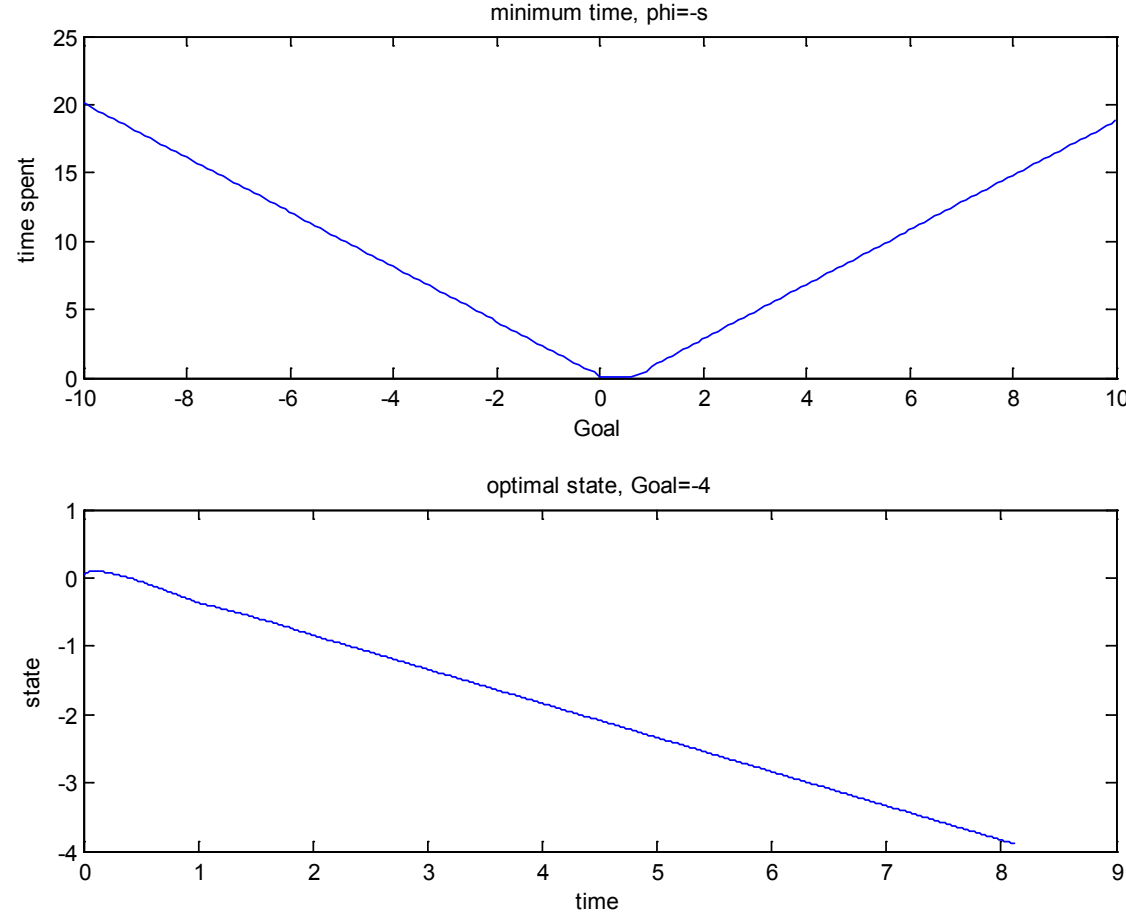

Figure 3.8

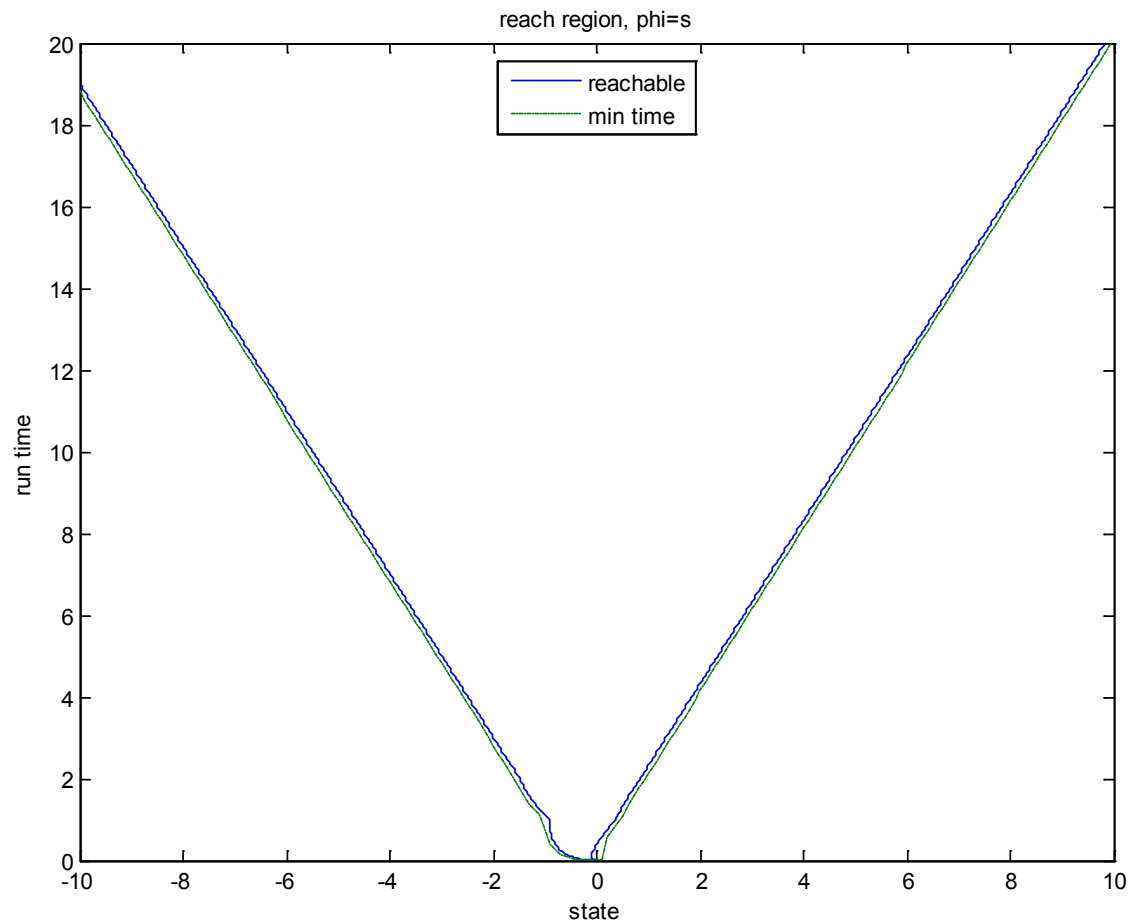

Figure 3.9 

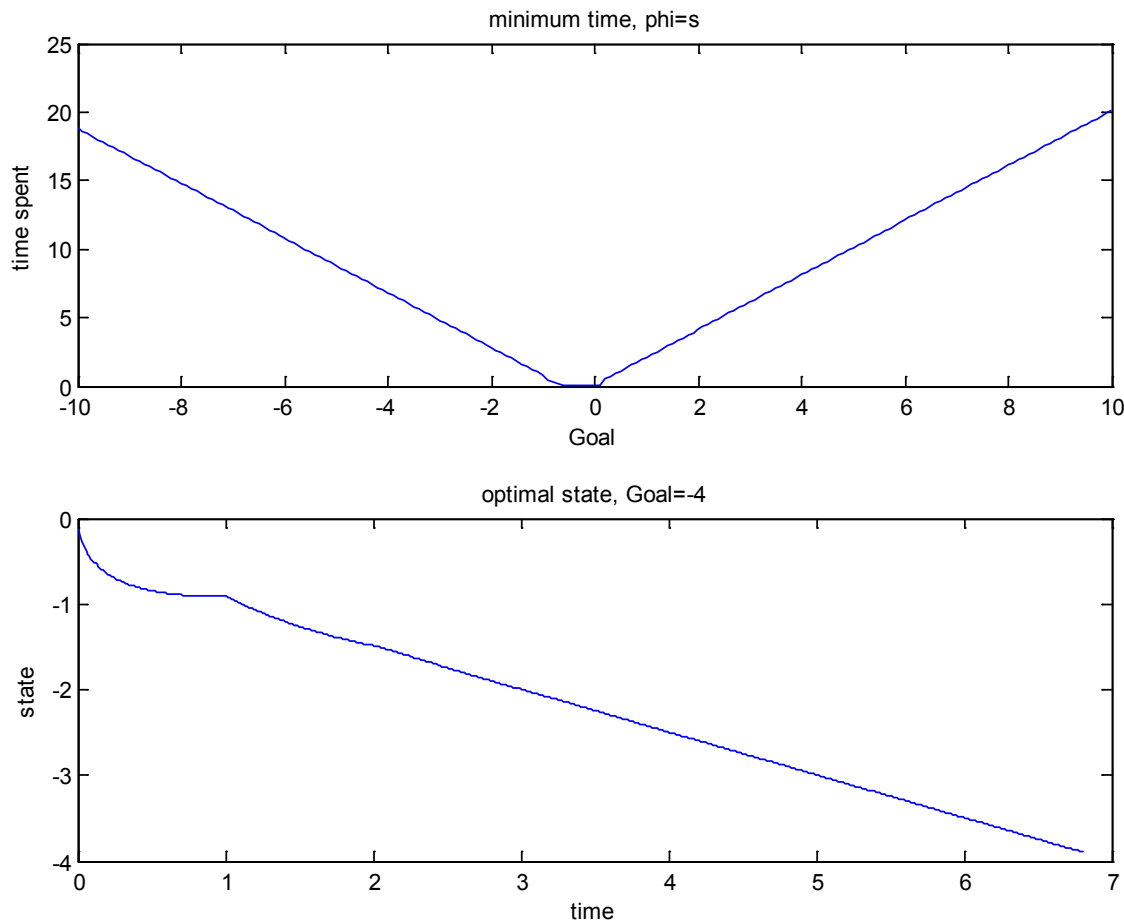

Figure 3.10

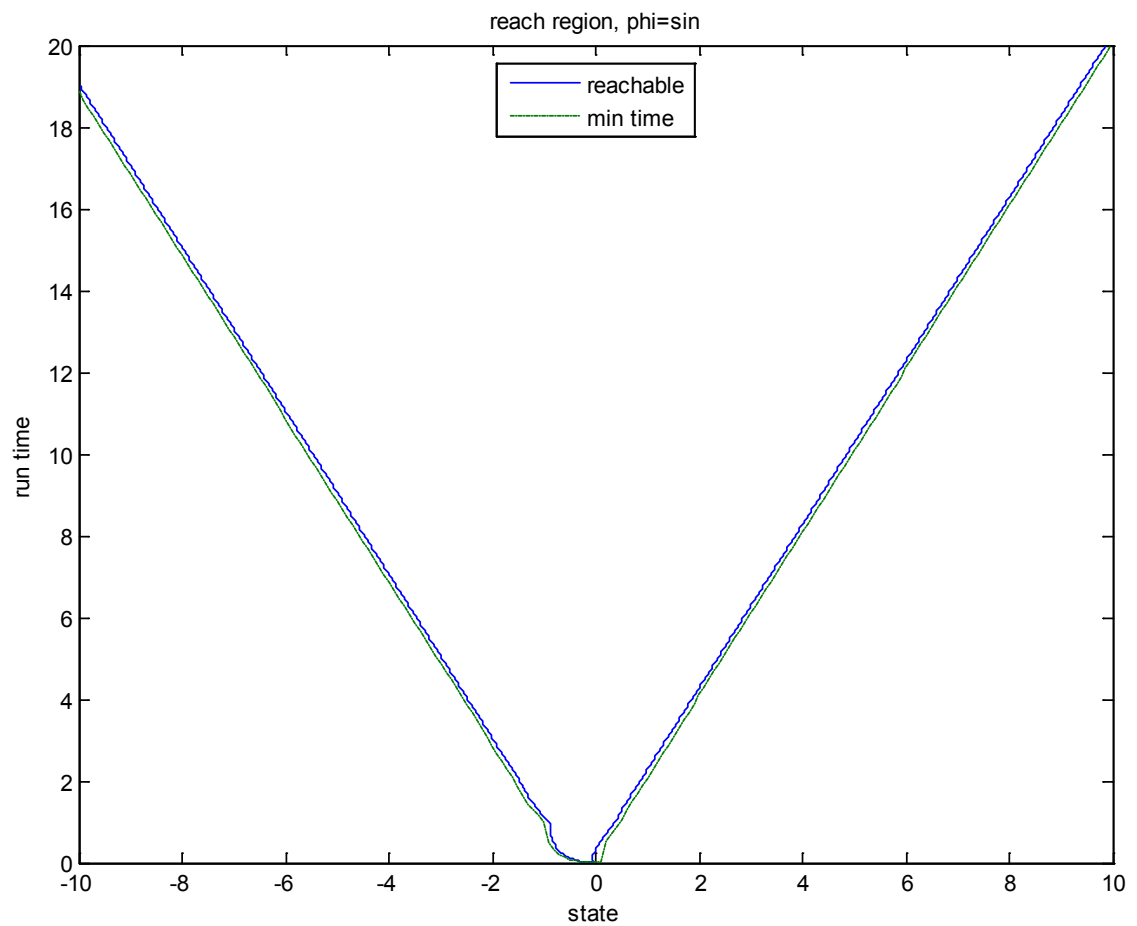

Figure 3.11 

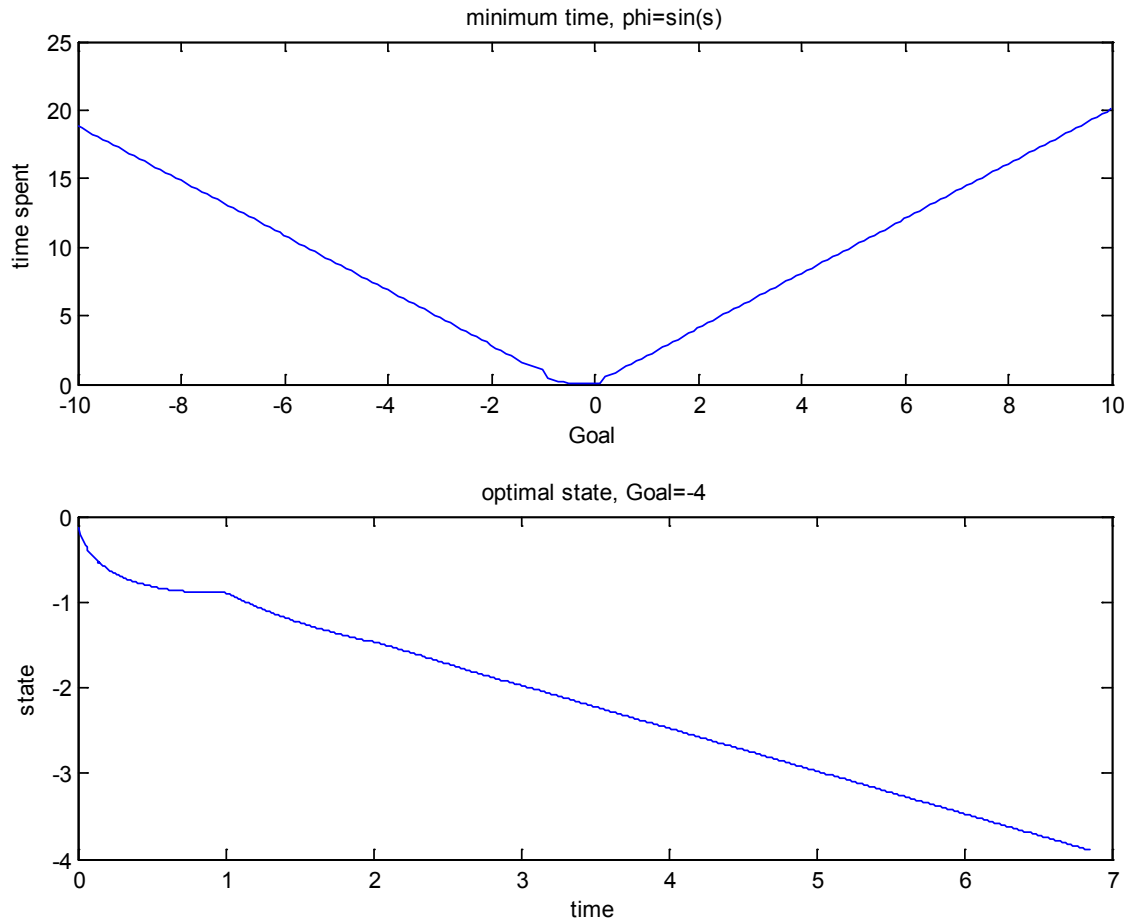

Figure 3.12

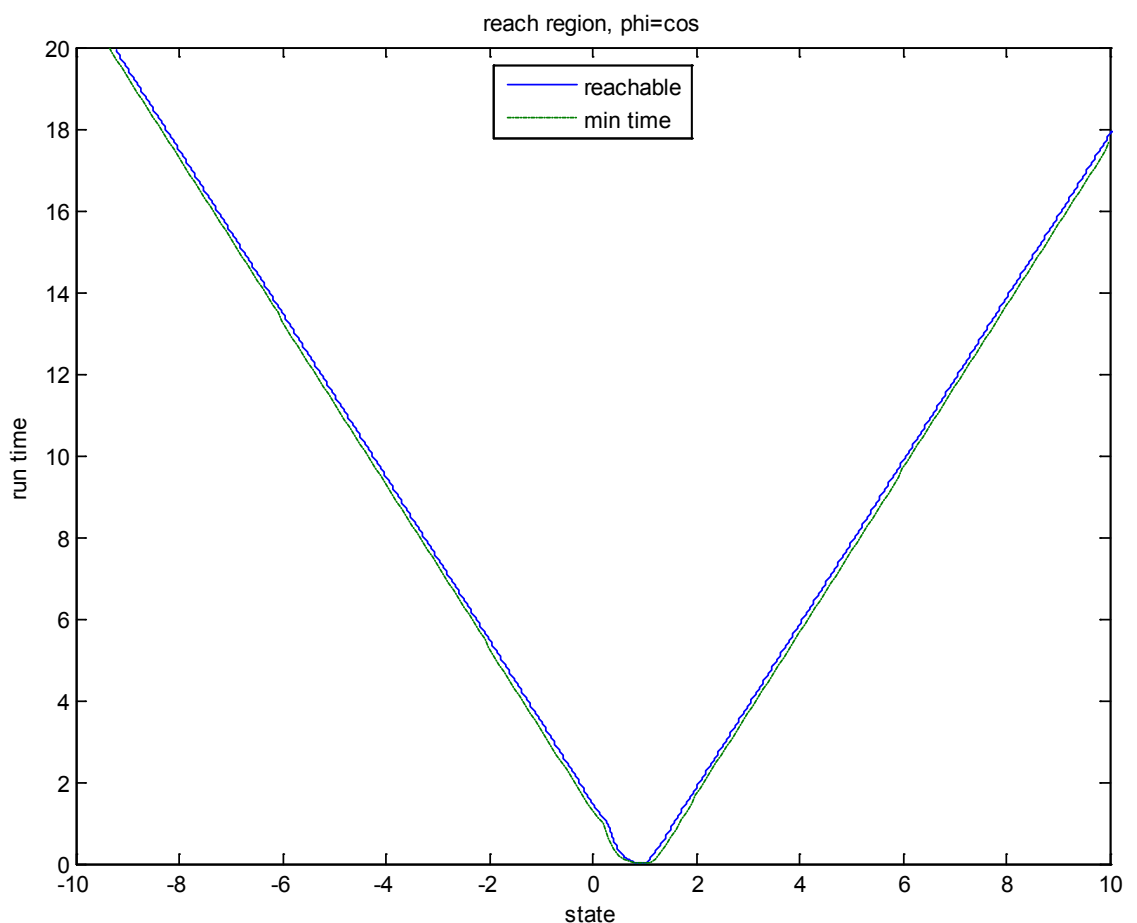

Figure 3.13 

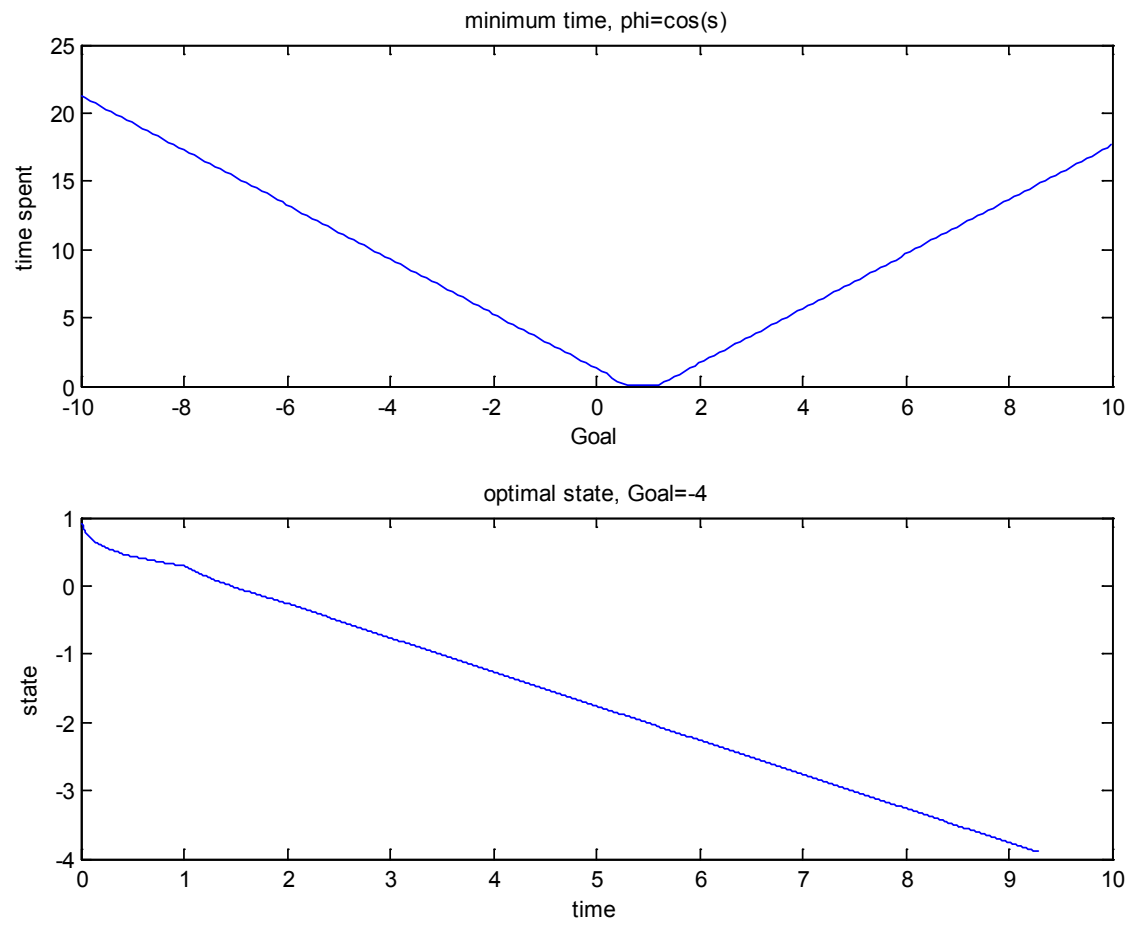

Figure 3.14

\section{References}

[1] J. A. Burns, E. M. Cliff and T. L. Herdman, A State-Space Model for an Aeroelastic System, Proceedings: $22^{\text {nd }}$ IEEE Conference on Decision and Control (1983) 1074-1077.

[2] Hsin-Hao Chen and Shihchung Chiang, A specific Procedure for Analytic Solutions to a Class of Singular Integral Equations, Chung Hua Journal of Science and Engineering, Vol.7, No. 3 (2009) 29-34.

[3] Shihchung Chiang, Notes on the Solution of a Class of Singular Integral Equations, Chung Hua Journal of Science and Engineering, Vol. 3, No. 4 (2005) 89-95.

[4] Shihchung Chiang, On the Numerical Solution of a Class of Singular Integro-Differential Equations, Chung Hua Journal of Science and Engineering, Vol.4, No. 3 (2006) 43-48.

[5] F. Kappel and K. P. Zhang, Equivalence of Functional Equations of Neutral Type and Abstract Cauchy Problems, Monatsch. Math. Vol. 101 (1986) 115-133.

[6] Chien-Chi Yu and Shihchung Chiang, On the Numerical
Optimal Controls for a Class of Integro-Differential Equations of Neutral Type, Chung Hua Journal of Computational Science, No. 1 (2011) 1-7.

[7] Shihchung Chiang, Numerical Optimal Issues to a Class of Neutral Singular Integro-Differential Equations, Chung Hua Journal of Computational Science, No. 2 (March, 2012) 7-17.

[8] Shihchung Chiang and Terry L. Herdman, Revised Numerical Methods for Optimal Control of a Class of Singular Integro-Differential Equations, Mathematics in Engineering, Science and Aerospace, Vol.4, No.2 (2013) 171-187.

[9] K. Ito and J. Turi, Numerical Methods for a class of Singular Integro-Differential Equations Based on Semigroup Approximation, SIAM J. Numer. Anal., Vol. 28, No. 6 (1991) 1698-1722.

[10] J. A. Burns and K. Ito, On Well-Posedness of Solutions to Integro-Differential Equations of Neutral-Type in a Weighted $L_{2}$ Spaces, Differential and Integral Equations, Vol. 8 (1995) 627-646. 